\title{
Topological Transition and Inductive Current Drive of a Translated Field-Reversed Configuration Plasma*)
}

\author{
Mamiko ARAI, Tomohiko ASAI, Seri KATAYAMA, Junpei ISHIWATA, Naoto ONO, \\ Junichi SEKIGUCHI, Tsutomu TAKAHASHI, Michiaki INOMOTO ${ }^{1)}$, Toshiki TAKAHASHI ${ }^{2)}$, \\ Shigefumi OKADA ${ }^{3)}$, Yoshiro NARUSHIMA ${ }^{4)}$ and Loren C. STEINHAUER ${ }^{5)}$ \\ College of Science and Technology, Nihon University, Tokyo 101-8308, Japan \\ ${ }^{1)}$ Graduate School of Frontier Sciences, The University of Tokyo, Chiba 277-8561, Japan \\ ${ }^{2)}$ Graduate School of Engineering, Gunma University, Gunma 376-0052, Japan \\ ${ }^{3)}$ Graduate School of Engineering, Osaka University, Osaka 565-0871, Japan \\ ${ }^{4)}$ National Institute for Fusion Science, Gifu 509-5292, Japan \\ ${ }^{5)}$ TAE Technologies, Inc., 19631 Pauling, Foothill Ranch, CA 92610, USA
}

(Received 28 December 2017 / Accepted 2 April 2018)

\begin{abstract}
A field-reversed configuration (FRC) is a high-beta compact toroid, ideally with an exclusive poloidal magnetic field. As an FRC has a simply-connected geometry, it can be translated along an external guide magnetic field and trapped in a confinement region with a quasi-static external magnetic field. In the FAT (FRC amplification via translation)-ICD (inductive current drive) experiment, a center solenoid is installed in a quasi-spherical confinement region to create an inductive toroidal current drive. An FRC formed by a field-reversed theta-pinch is translated at a velocity of $100-200 \mathrm{~km} / \mathrm{s}$ into a confinement region comprising a center structure. Such an FRC then changes from a simply-connected structure to a torus. The magnetic field structures of FRCs have been directly observed using an internal magnetic probe array installed at the mid-plane of the confinement region. We observed that an FRC can be translated successfully without any disruptive perturbation to its structure and that the magnetic flux can be quadrupled via the inductive current drive, as compared with the cases without an inductive current drive. Herein, we report in detail the evidence for the inductive drive and relaxation process of the translated FRC.
\end{abstract}

(C) 2018 The Japan Society of Plasma Science and Nuclear Fusion Research

Keywords: field-reversed configuration, center solenoid, inductive current drive, field-reversed theta-pinch

DOI: $10.1585 /$ pfr. 13.3402078

\section{Introduction}

A field-reversed configuration (FRC) is a high-beta $(\langle\beta\rangle \sim 0.9)$, compact toroid, ideally with a poloidal magnetic field [1]. Owing to its extremely high-beta nature i.e. low magnetic field, the most efficient technique for producing additional heating of an FRC is the neutral-beam injection; however, an FRC formed by a field-reversed thetapinch (FRTP) [2], which is the most effective way to form an FRC, may not have sufficient trapped poloidal flux to capture the tangentially injected, fast ion beams.

As an FRC has a simply connected magnetic configuration, it can be translated axially along the gradient of an external guide magnetic field and trapped in a confinement region with a static external magnetic field. The translated FRC initially reveals a modest amount of toroidal flux, as observed at the FRX-C/T [3], FIX [4], and TCS facilities [5]. The TCS experiment demonstrated self-organization of a translated FRC, thereby converting the FRC into a spherical tokamak type of magnetic configuration. This

author's e-mail: asai.tomohiko@nihon-u.ac.jp

*) This article is based on the presentation at the 26th International Toki Conference (ITC26). spontaneously generated toroidal flux may decrease the beta value; however, such a flux can potentially maintain the radial force-balance, even with a center structure on the geometrical axis of the confinement chamber. Moreover, electrons can flow along the toroidal magnetic field lines, driven by the induced azimuthal electric field.

The FRC Amplification via Translation (FAT) facility at Nihon University, Tokyo, Japan, was built to study the behavior of a translated FRC, in particular, the transition of its magnetic structure during translation into a large-bore confinement region [6]. The FAT has vacuum vessels made of transparent quartz tubes in both the formation and confinement regions. The formation chamber is surrounded by a one-turn theta-pinch coil made of cast copper, and the confinement chamber is surrounded by a multilayered coil. The bobbin of the multilayered coil is made of stainless steel, which conserves magnetic flux on the timescale of the translation of the FRC. The theta-pinch coil generates a bias field of $\sim 0.05 \mathrm{~T}$, following which a reversed field of $\sim 0.6 \mathrm{~T}$ is applied rapidly (with a rise time of $\sim 4 \mu \mathrm{s}$ ) in the formation region. The generated FRTP-FRC 
is translated from the formation region into the confinement region along the gradient of a guide magnetic field of $\sim 0.1$ T. Despite this dramatic change, the translated FRC reaches a quiescent phase without being disrupted.

In the FAT device, typical plasma parameters are: separatrix radius $r_{\Delta \phi} \sim 0.06 \mathrm{~m}$, length $l_{\Delta \phi} \sim 1.0 \mathrm{~m}$, ion temperature $T_{\mathrm{i}} \sim 100 \mathrm{eV}$, and electron density $n_{\mathrm{e}} \sim 3 \times 10^{21} \mathrm{~m}^{-3}$. Moreover, a typical translated FRC has a separatrix radius $r_{\Delta \phi} \sim 0.2 \mathrm{~m}$, length $l_{\Delta \phi} \sim 0.7 \mathrm{~m}$, and ion temperature $T_{\mathrm{i}} \sim$ $40 \mathrm{eV}$. The FRTP-FRC is translated at a velocity comparable to the Alfvén velocity $(100-200 \mathrm{~km} / \mathrm{s})$ into the confinement region.

\section{Experimental Setup}

A schematic diagram of the FAT facility (FAT-ICD) with a center solenoid (CS) is presented in Fig. 1 (a). The FRTP-FRC is translated at $100-200 \mathrm{~km} / \mathrm{s}$ along the external guide magnetic field into the confinement region. Herein, the FRC is pierced along its geometric axis by a "cantilevered" CS present in a stainless steel housing called the "liner." The liner works as a conducting shell to provide global stability to the translated FRC [7, 8]. In this series of experiments, the CS was installed on the geometrical axis of the quasi-spherical confinement region. Table 1 lists the specifications of the CS.

The CS comprises a double-layered solenoid. Each solenoidal layer comprises a spirally machined aluminum pipe, as presented in Fig. 1 (b). The solenoid is mechanically reinforced by carbon-fiber-reinforced plastic (CFRP) pipes and is held within the stainless steel exoskeleton to maintain the vacuum state of the chamber. The CS is discharged when the translating FRC reaches the mid-plane $(z=0)$ in the confinement region $(t=30 \mu \mathrm{s}$; the formation of an FRTP-FRC is triggered at $t=0$ ). The current through

Table 1 Specifications of the center solenoid.

\begin{tabular}{|c|c|c|c|c|c|}
\hline Name & Material & $\begin{array}{l}\text { I.D. } \\
(\mathrm{mm})\end{array}$ & $\begin{array}{l}\text { Thickness } \\
(\mathrm{mm})\end{array}$ & $\begin{array}{l}\text { Length } \\
(\mathrm{mm})\end{array}$ & $\begin{array}{c}\text { Turn } \\
\text { Number }\end{array}$ \\
\hline Liner & SUS304 & 60.5 & 0.3 & 2100 & - \\
\hline \multicolumn{2}{|c|}{ CFRP } & 58.9 & 1.6 & 2240 & - \\
\hline CS-out & $\mathrm{Al}$ & 55.0 & 3.0 & 2300 & 45 \\
\hline CS-in & $\mathrm{Al}$ & 45.0 & 3.0 & 2500 & 45 \\
\hline \multicolumn{2}{|c|}{ CFRP } & 37.8 & 1.6 & 2600 & - \\
\hline
\end{tabular}

(a) $r(m)$

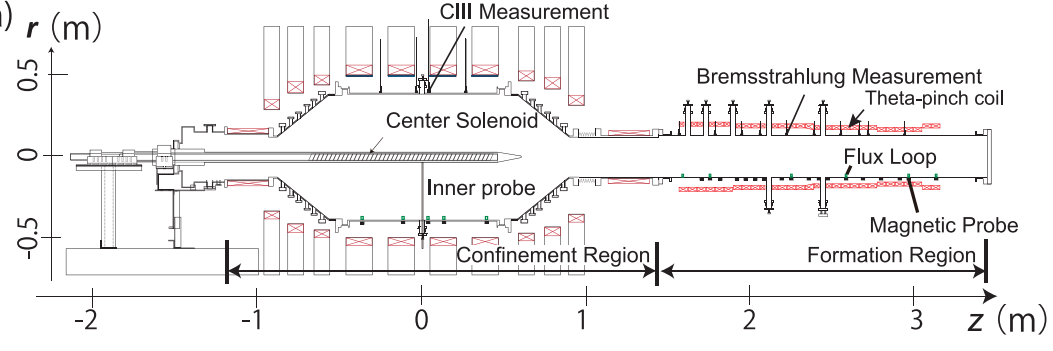

the CS is $\sim 24 \mathrm{kA}$, and its rise time is $\sim 100 \mu \mathrm{s}$.

In the confinement region, five sets of magnetic probes and one turn flux loops are mounted on the quartz tube to measure the axial magnetic field and magnetic flux. The excluded flux radius $\left(r_{\Delta \phi}\right)$, which represents the separatrix radius, is inferred by the excluded flux method. Internal magnetic-probe arrays at the mid-plane of the confinement region measure the internal poloidal and toroidal magnetic field profiles in the translated FRC.

\section{Inductive Current Drive}

The radial profiles of poloidal magnetic field with and without the inductive current drive, as measured at the midplane of the confinement region by the internal magneticprobe array, are compared in Fig. 2. The blue and red points represent the results with and without discharging through the CS, respectively. The red points indicate the reversal of the internal magnetic field within $r \sim 0.2 \mathrm{~m}$. This demonstrates that the FRTP-FRC was successfully translated into the confinement region comprising the CS without any disruptive perturbation to its magnetic structure. The stainless steel exoskeleton layer (liner) supports the inboard side plasma pressure without any adverse effect

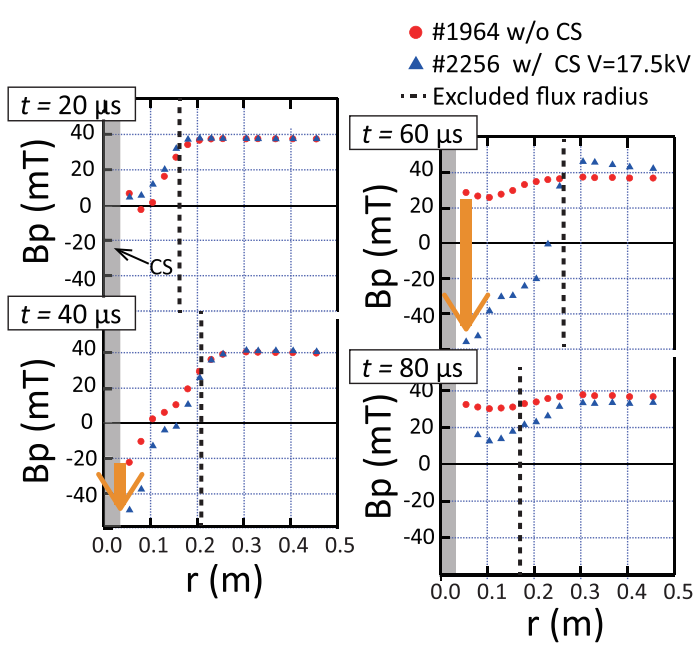

Fig. 2 Time evolution of the radial profile of the poloidal magnetic field $(z=0)$.

(b)

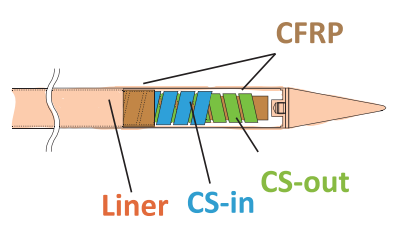

Fig. 1 Schematic views of (a) the FAT-ICD device, with the positions of the magnetic probe, flux loops, and installed internal magneticprobe array indicated, and (b) the internal structure of the center solenoid (CS); see text for definitions of symbols. 

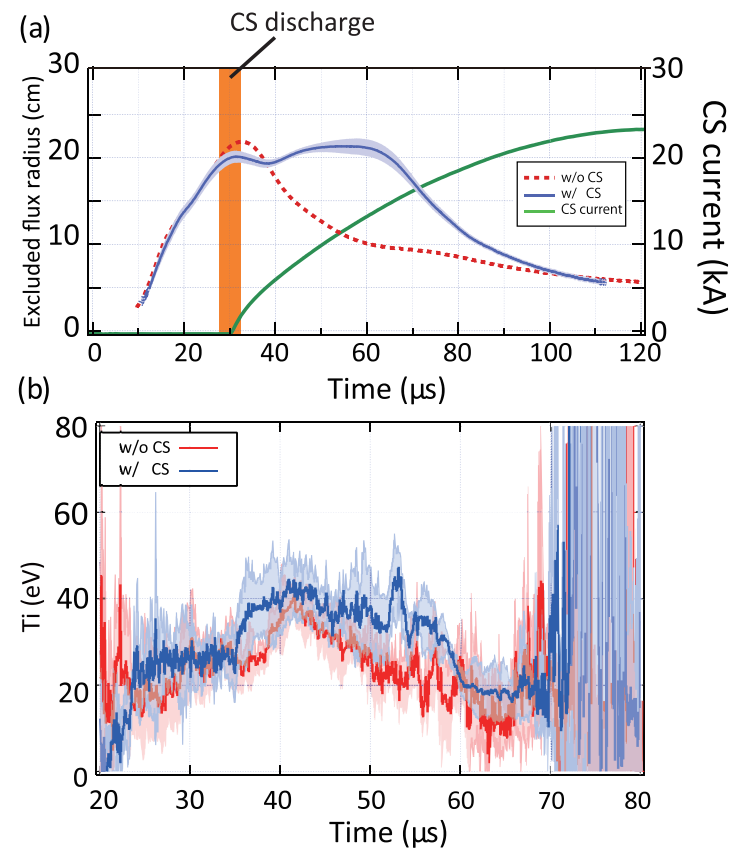

Fig. 3 Time evolution of the (a) excluded-flux radius, together with the discharge waveform and (b) ion temperature at the mid-plane of the confinement region. The shaded areas represent the standard deviations.

on the plasma. When the current is activated in the CS, the internal poloidal magnetic field increases, compared with the case without the CS discharge, as indicated by the blue points in Fig. 2. The internal magnetic field peaks at $58 \mathrm{mT}$ with the inductive current drive. This is approximately 2.6 times higher than that of the discharge without activating the CS.

Figure 3 (a) presents the time evolution of the excluded-flux radius of the translated FRC at the mid-plane of the confinement region, collectively with the discharge current through the CS (solid green line). The solid blue and broken red lines are the results with and without the $\mathrm{CS}$, respectively. In both cases, the excluded flux radius is approximately $20 \mathrm{~cm}$ just after translation. Without an activated CS, the excluded flux immediately begins to decay; however, when the CS is activated, the excluded flux radius is sustained at a roughly constant level for about $30 \mu \mathrm{s}$ longer before beginning to decay. Figure 3 (b) presents the time evolution of the ion temperature with and without the current drive. Translation into the CS area does not cause any significant temperature decrease. After the discharge through the CS, the case with the CS reveals higher ion temperature with the maximum difference being about $30 \%(t \sim 50 \mu \mathrm{s})$.

Figure 4 presents the axial profile of the excluded-flux radius with the inductive current drive in the confinement region. For these experiments, the translated FRC exhibits axial bouncing motions after reflection at the conical ends of the metal chamber; however, the FRC remains within the area of the CS.
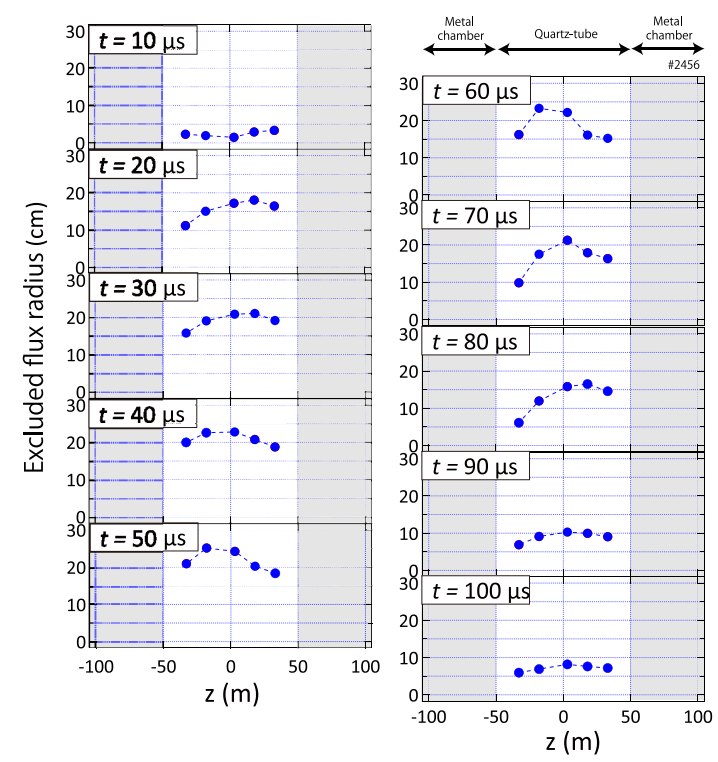

Fig. 4 Time evolution of the axial profile of the excluded-flux radius in the confinement region with the inductive current drive.

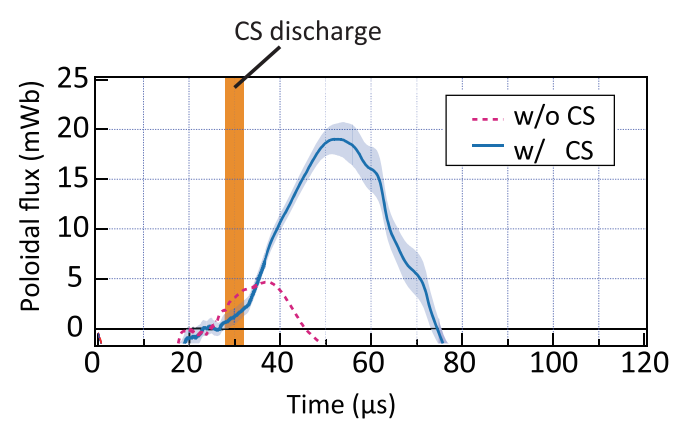

Fig. 5 Time evolution of the poloidal-flux radius at the midplane of the confinement region.

The time evolution of the trapped poloidal magnetic flux, as measured with the internal magnetic probe, is presented in Fig. 5. The blue and red lines represent cases with and without the inductive current drive, respectively. The internal poloidal magnetic probe signals at the mid-plane of the confinement region, averaged over several FRCs with the same discharge conditions, are presented in Figs. 2 and 3. Typical standard deviations are indicated. Without discharging through the CS, the magnetic flux is approximately $5 \mathrm{mWb}$, and it decays rapidly. With the discharge, the magnetic flux is $\sim 18 \mathrm{mWb}$, and it maintains this value up to approximately $t \sim 60 \mu \mathrm{s}$. The associated excludedflux radius similarly maintains a roughly constant value over the same time period.

Figure 6 presents the dependence of the (a) excluded flux radius and (b) poloidal magnetic flux through the inductive current drive wherein both depend linearly on the temporal change of the magnetic flux generated by the CS. 

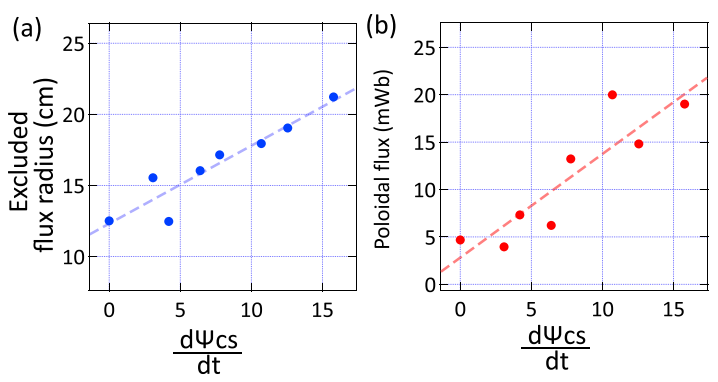

Fig. 6 Dependence of the (a) excluded-flux radius and (b) poloidal flux of the translated FRC on the inductive toroidal current.
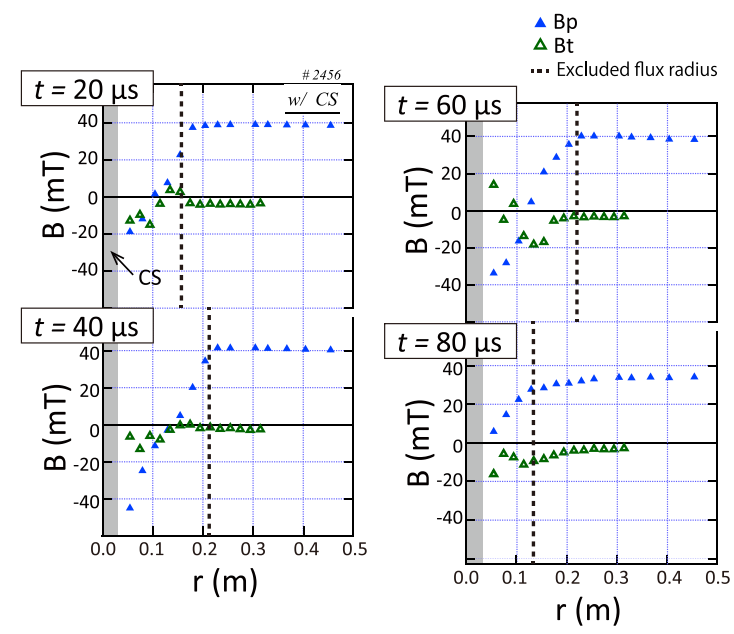

Fig. 7 Time evolution of the radial profile of the poloidal and toroidal magnetic fields $(z=0$; charging voltage of the CS is $17.5 \mathrm{kV}$ ).

\section{Discussion}

The experimental results reveal that the inductive toroidal current drive has been achieved successfully with the CS. Simultaneously, the internal probe measurements indicate that the toroidal flux is spontaneously generated both with and without the inductive current drive during and after the translation process, with no externally applied poloidal current. This is consistent with previous FRC translation studies. The toroidal current applied through the CS induces an azimuthal electric field that drives electrons along the magnetic field lines. Consequently, the poloidal magnetic flux is increased by the inductive toroidal current drive, whereas the toroidal magnetic component also increases. The induced toroidal mag- netic field is about one-third of the poloidal magnetic field and is in the same direction as the current through the CS. In the latter phase of the discharge, the magnetic profile of the plasma relaxes toward a spheromak-like configuration, as presented in Fig. 7. Further studies are required to understand this relaxation process.

To obtain the inductive current drive, the CS discharge must begin when the FRC is translated to the mid-plane of the confinement region. If the CS discharges before the FRC reaches the mid-plane of the confinement region, the excluded-flux radius does not increase.

\section{Summary}

Toroidal current drive was applied to an FRC that has been translated into a quasi-spherical confinement region. The FRTP-FRC was successfully translated without any disruptive perturbation to its magnetic structure. The poloidal magnetic flux was increased by discharging the CS.

The experimental results reveal successful inductive toroidal current drive of FRCs. This method has significant implications as an effective tool for improving the performance of an FRTP-FRC, in particular, to provide a suitable target for additional heating techniques, such as high-energy neutral beam injection.

\section{Acknowledgments}

The authors would like to thank the past and present members of our laboratory. This work was partially supported by JSPS KAKENHI Grant Number 16K06939 and the NIFS Collaborative Research Program (NIFS17KN WP006).

[1] M. Tuszewski, Nucl. Fusion 28, 2033 (1988).

[2] Ts. Takahashi and Y. Hirano, J. Plasma Fusion Res. 84, 507 (2008).

[3] M. Tuszewski and B.L. Wright, Phys. Rev. Lett. 63, 2236 (1989).

[4] A. Shiokawa and S. Goto, Phys. Fluids B 5, 534 (1993).

[5] H.Y. Guo, A.L. Hoffman, L.C. Steinhauer and K.E. Miller, Phys. Rev. Lett. 95, 175001 (2005).

[6] J. Sekiguchi, T. Asai and Ts. Takahashi, Rev. Sci. Instrum. 89, 013506 (2017).

[7] Y. Ono, T. Akao, M. Katsurai and M. Yamada, Phys. Fluids B 5, 3691 (1993).

[8] S.P. Gerhardt, E. Belova, M. Inomoto et al., Phys. Plasmas 13, 112508 (2006). 